\title{
Establishing the existence of Hilfer fractional pantograph equations with impulses
}

\author{
Sugumaran Harikrishnan ${ }^{\mathrm{a}}$, Rabha W. Ibrahim ${ }^{\mathrm{b} *}$ and Kuppusamy Kanagarajan ${ }^{\mathrm{a}}$ \\ ${ }^{a}$ Department of Mathematics, Sri Ramakrishna Mission Vidyalaya College of Arts and Science, Coimbatore-641020, India \\ ${ }^{\mathrm{b}}$ Faculty of Computer Science and Information Technology, , University Malaya, 50603, Malaysia \\ *Corresponding author E-mail: rabhaibrahim@yahoo.com
}

\section{Article Info}

Keywords: Pantograph equation, Impulsive condition, Fixed point stability 2010 AMS: 26A33, 34K40, 34K14

Received: 16 March 2018

Accepted: 17 April 2018

Available online: 30 June 2018

\begin{abstract}
In [1], the authors established the existence of a class of fractional differential equations of a complex order. In this note, we derive some sufficient conditions for the existence of solutions to a class of Hilfer fractional pantograph equations with impulsive effect. Further, using the techniques of nonlinear functional analysis, we establish appropriate conditions and results to discuss various kinds of Ulam-Hyers stability.
\end{abstract}

\section{Preface}

Fractional calculus is an emerging field in applied mathematics that deals with derivatives and integrals of arbitrary orders. For their applications and details note, one can refer to $[8,13,17]$. Due to the properties involved in Hilfer fractional type of derivatives (introduced by Hilfer [8]) in the sense that it generalizes the Riemann-Liouville (R-L) and Liouville-Caputo (L-C) fractional derivatives, a lot of studies have been done on it, including the existence and uniqueness of solutions to such differential equations (DEs) involving Hilfer fractional derivative (HFD); see [4, 7, 20, 21], and references therein.

It is well known that the pantograph equations (PEs) arises in quite different fields of pure and applied mathematics and have been investigated extensively. Recently, due to its importance in many applied fields and playing an extremely important role in explaining many different phenomena, for details see $[3,6,10,19,22]$.

Recently impulsive DEs have been considered by many authors due to their significant applications in various fields of science and technology. For detail study, see $[2,14,15,16,18,24]$. Due to its large number of applications, this area has been received great importance and remarkable attention from the researchers.

In 1940, Ulam posed the following problem about the stability of functional equations: Under what conditions does there exist an additive mapping near an approximately additive mapping? In the following year, Hyers gave an answer to the problem of Ulam for additive functions defined on Banach spaces, [8]. That is why the name of this stability is Ulam-Hyers (U-H) stability. Later on, Hyers results are extended by many mathematicians. The stability analysis is extremely helpful in numerous applications, for example, numerical analysis and optimization, where it is very tough to find the exact solution of a nonlinear problem. The aforementioned stability has very recently attracted the attention of researchers; we refer the reader to some papers [11, 12,23]. Because of, fractional order system may have more attractive feature over the integer order system.

Consider the PEs with impulsive condition given by

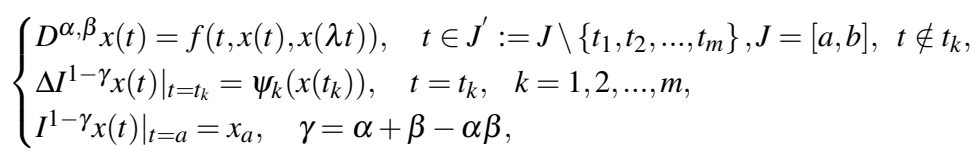

where $D^{\alpha, \beta}(0<\alpha<1,0 \leq \beta \leq 1)$ is the Hilfer fractional derivative of orders $\alpha$ and type $\beta$. Here, $0<\lambda<1$ the function $f: J \times R \times R \rightarrow R$ is

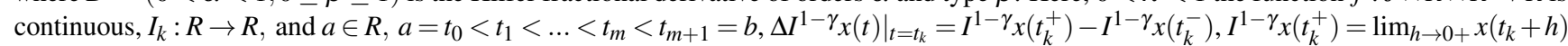

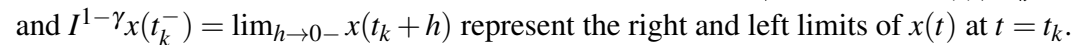


The paper is organized as follows: In Section 2, we present some necessary definitions and preliminary results that will be used to prove our main results. The proofs of soultion existence and uniqueness are given in Section 3. Finally, stability is proved in Section 4.

\section{Prerequisites}

In this section, we recall some preliminaries materials required in this paper from. Consider the following space

$$
P C[J, R]=\left\{x: J \rightarrow R: x(t) \in C\left(t_{k}, t_{k+1}\right], k=0, \ldots, m \text {; there exists } x\left(t_{k}^{+}\right) \text {and } x\left(t_{k}^{-}\right)\right\} .
$$

Now we consider the weighted space $P C_{\gamma}[J, R]$,

$$
P C_{\gamma}[J, R]=\left\{x:\left.(t-a)^{\gamma} x(t)\right|_{t \in\left[t_{k}, t_{k+1}\right]} \in C\left[t_{k}, t_{k+1}\right], k=0, \ldots, m \text { where } 0 \leq \gamma<1\right\} .
$$

Obviously, which is a Banach space with norm

$$
\|x\|_{P C_{\gamma}}=\sup _{t \in\left(t_{k}, t_{k+1}\right]}\left\{\left(t-t_{k}\right)^{\gamma} x(t)\right\}, k=0, \ldots, m .
$$

Definition 2.1. [13] The $R$ - L fractional integral of order $\alpha>0$ of function $f:[0, \infty) \rightarrow R$ can be written as

$$
I^{\alpha} f(t)=\frac{1}{\Gamma(\alpha)} \int_{0}^{t}(t-s)^{\alpha-1} f(s) d s
$$

Definition 2.2. [13] The R-L fractional derivative of order $\alpha>0$ of a continuous function $f:[0, \infty) \rightarrow R$ can be written as

$$
D^{\alpha} f(t)=\frac{1}{\Gamma(n-\alpha)}\left(\frac{d}{d t}\right)^{n} \int_{0}^{t}(t-s)^{\alpha-n+1} f(s) d s,
$$

provided that the right side is pointwise defined on $[0, \infty)$.

Definition 2.3. [13] The L-C fractional derivative of order $\alpha>0$ of a continuous function $f:[0, \infty) \rightarrow R$ can be written as

$$
{ }^{C} D^{\alpha} f(t)=D^{\alpha}\left[f(t)-\sum_{k=0}^{n-1} \frac{t^{k}}{k !} f^{k}(0)\right], t>0, n-1<\alpha<n .
$$

Definition 2.4. [8] The HFD of order $0<\alpha<1$ and $0 \leq \beta \leq 1$ of function $f(t)$ is defined by

$$
D^{\alpha, \beta} f(t)=\left(I^{\beta(1-\alpha)} D\left(I^{(1-\beta)(1-\alpha)} f\right)\right)(t) .
$$

The GRL fractional derivative is considered as an interpolation between the $R$ - $L$ and $L-C$ fractional derivative and the relations are given below.

Remark 2.5. (i) Operator $D^{\alpha, \beta}$ also can be written as

$$
D^{\alpha, \beta}=\left(I^{\beta(1-\alpha)} D\left(I^{(1-\beta)(1-\alpha)}\right)\right)=I^{\beta(1-\alpha)} D^{\gamma}, \quad \gamma=\alpha+\beta-\alpha \beta .
$$

(ii) If $\beta=0$, then $D^{\alpha, \beta}=D^{\alpha, 0}$ is called $R$ - $L$ fractional derivative.

(iii) If $\beta=1$, then $D^{\alpha, \beta}=I^{1-\alpha} D$ is called $L$-C fractional derivative.

Lemma 2.6. [4] If $\alpha>0$ and $\beta>0$, there exists

$$
\left[I^{\alpha}(t)^{\beta-1}\right](x)=\frac{\Gamma(\beta)}{\Gamma(\beta+\alpha)} x^{\beta+\alpha-1},
$$

and

$$
\left[D^{\alpha}(t)^{\alpha-1}\right](x)=0, \quad 0<\alpha<1 .
$$

Lemma 2.7. [4] If $\alpha>0$ and $\beta>0$ and $f \in L^{1}(a, b]$, there exists the following properties

$$
I^{\alpha} I^{\beta} f(t)=I^{\alpha+\beta} f(t),
$$

and

$$
D^{\alpha} I^{\alpha} f(t)=f(t) .
$$

Lemma 2.8. [4] Let $\gamma=\alpha+\beta-\alpha \beta$ where $0<\alpha<1$ and $0 \leq \beta \leq 1$. Let $f: J \times R \rightarrow R$ be a function such that $f(\cdot,(\cdot)) \in C_{1-\gamma}[a, b]$ for any $x \in C_{1-\gamma}[a, b]$. If $x \in C_{1-\gamma}^{\gamma}[a, b]$, then $x$ satisfies

$$
\begin{aligned}
D^{\alpha, \beta} x(t) & =f(t, x(t)), \quad t \in(a, b] \\
I^{1-\gamma_{x}(a)} & =x_{a} .
\end{aligned}
$$

if and only if $x$ satisfies

$$
x(t)=\frac{x_{a}}{\Gamma(\gamma)}(t-a)^{\gamma-1}+\frac{1}{\Gamma(\alpha)} \int_{a}^{t}(t-s)^{\alpha-1} f(s, x(s)) d s
$$


Lemma 2.9. Let $f: J \rightarrow R$ be continuous. A function $x \in P C_{1-\gamma}[J, R]$ is a solution of the fractional differential equation

$$
\begin{aligned}
& D^{\alpha, \beta} x(t)=f(t), \quad t \in J^{\prime} \\
& I^{1-\gamma} x\left(t_{i}\right)=x_{t_{i}},
\end{aligned}
$$

if and only if $x$ is a solution of the integral equation

$$
x(t)=\frac{x_{t_{i}}}{\Gamma(\gamma)}(t-a)^{\gamma-1}-\frac{(t-a)^{\gamma-1}}{\Gamma(\gamma) \Gamma(1-\beta(1-\alpha))} \int_{a}^{t_{i}}\left(t_{i}-s\right)^{(1-\beta(1-\alpha))-1} f(s) d s+\frac{1}{\Gamma(\alpha)} \int_{a}^{t}(t-s)^{\alpha-1} f(s) d s .
$$

Next, we shall give the definitions and the criteria of U-H stability and U-H-R stability for PEs with impulsive effect under Hilfer fractional derivative. Let $\varepsilon$ be a positive number and $\varphi: J \rightarrow R^{+}$be a continuous function, for every $t \in J^{\prime}$ and $k=1,2, \ldots, m$, we have the following inequalities

$$
\begin{aligned}
& \left\{\begin{array}{l}
\left|D^{\alpha, \beta} z(t)-f(t, z(t), z(\lambda t))\right| \leq \varepsilon \\
\left.\Delta I^{1-\gamma} z(t)\right|_{t=t_{k}}-\psi_{k}\left(z\left(t_{k}\right)\right) \mid \leq \varepsilon
\end{array}\right. \\
& \left\{\begin{array}{l}
\left|D^{\alpha, \beta} z(t)-f(t, z(t), z(\lambda t))\right| \leq \varepsilon \varphi(t), \\
\Delta I^{1-\left.\gamma_{z}(t)\right|_{t=t_{k}}-\psi_{k}\left(z\left(t_{k}\right)\right) \mid} \leq \varepsilon \varphi(t),
\end{array}\right. \\
& \left\{\begin{array}{l}
\left|D^{\alpha, \beta} z(t)-f(t, z(t), z(\lambda t))\right| \leq \varphi(t), \\
\left.\Delta I^{1-\gamma} z(t)\right|_{t=t_{k}}-\psi_{k}\left(z\left(t_{k}\right)\right) \mid \leq \varphi(t),
\end{array}\right.
\end{aligned}
$$

Definition 2.10. Eq. (1.1) is $U$-H stable if there exists a real number $C_{f}>0$ such that for each $\varepsilon>0$ and for each solution $z \in P C_{1-\gamma}[J, R]$ of the inequality (2.3) there exists a solution $x \in P C_{1-\gamma}[J, R]$ of Eq. (1.1) with

$$
|z(t)-x(t)| \leq C_{f} \varepsilon, \quad t \in J .
$$

Definition 2.11. Eq. (1.1) is generalized $U$-H stable if there exist $\varphi \in P C_{1-\gamma}\left[J, R^{+}\right], \varphi_{f}(0)=0$ such that for each solution $z \in P C_{1-\gamma}[J, R]$ of the inequality (2.3) there exists a solution $x \in P C_{1-\gamma}[J, R]$ of Eq. (1.1) with

$$
|z(t)-x(t)| \leq \varphi_{f} \varepsilon, \quad t \in J .
$$

Definition 2.12. Eq. (1.1) is U-H-R stable with respect to $\varphi \in P C_{1-\gamma}\left[J, R^{+}\right]$if there exists a real number $C_{f}>0$ such that for each solution $z \in P C_{1-\gamma}[J, R]$ of the inequality (2.4) there exists a solution $x \in P C_{1-\gamma}[J, R]$ of Eq. (1.1) with

$$
|z(t)-x(t)| \leq C_{f} \varepsilon \varphi(t), \quad t \in J .
$$

Definition 2.13. Eq. (1.1) is generalized $U-H-R$ stable with respect to $\varphi \in P C_{1-\gamma}\left[J, R^{+}\right]$if there exists a real number $C_{f, \varphi}>0$ such that for each solution $z \in P C_{1-\gamma}[J, R]$ of the inequality (2.5) there exists a solution $x \in P C_{1-\gamma}[J, R]$ of Eq. (1.1) with

$$
|z(t)-x(t)| \leq C_{f, \varphi} \varphi(t), \quad t \in J .
$$

Remark 2.14. A function $z \in P C_{1-\gamma}[J, R]$ is a solution of the inequality

$$
\left|D^{\alpha, \beta} z(t)-f(t, z(t), z(\lambda t))\right| \leq \varepsilon
$$

if and only if there exist a function $g \in P C_{1-\gamma}[J, R]$ and a sequence $g_{k}, k=1,2, \ldots, m$ (which depend on $z$ ) such that

(i) $|g(t)| \leq \varepsilon,\left|g_{k}\right|<\varepsilon$.

(ii) $D^{\alpha, \beta} z(t)=f(t, z(t), z(\lambda t))+g(t)$.

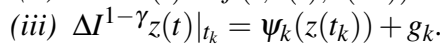

(iv) Let $0<\alpha<1,0 \leq \beta \leq 1$, if $z$ is solution of the inequality (2.3) then $z$ is a solution of the following integral inequality

$$
\left|z(t)-\frac{x_{a}}{\Gamma(\gamma)}(t-a)^{\gamma-1}-\frac{\sum_{0<t_{k}<t} \psi_{k}\left(z\left(t_{k}\right)\right)}{\Gamma(\gamma)}(t-a)^{\gamma-1}-\frac{1}{\Gamma(\alpha)} \int_{a}^{t}(t-s)^{\alpha-1} f(s, z(s), z(\lambda s)) d s\right| \leq \varepsilon\left(m+\frac{(b-a)^{\alpha}}{\Gamma(\alpha+1)}\right) .
$$

Lemma 2.15. [25] Let $a(t)$ be a nonnegative function locally integrable on $a \leq t<b$ for some $b \leq \infty$, and let $g(t)$ be a nonnegative, nondecreasing continuous function defined on $a \leq t<b$, such that $g(t) \leq K$ for some constant $K$. Further let $x(t)$ be a nonnegative locally integrable on $a \leq t<b$ function satisfying

$$
|x(t)| \leq a(t)+g(t) \int_{a}^{t}(t-s)^{\alpha-1} x(s) d s, t \in[a, b)
$$

with some $\alpha>0$. Then

$$
|x(t)| \leq a(t)+\int_{a}^{t}\left[\sum_{n=1}^{\infty} \frac{(g(t) \Gamma(\alpha))^{n}}{\Gamma(n \alpha)}(t-s)^{n \alpha-1}\right] a(s) d s, a \leq t<b .
$$


Remark 2.16. Under the hypethesis of Lemma 2.15 let a $(t)$ be a nondecreasing function on $[0, T)$. Then $y(t) \leq a(t) E_{\alpha}\left(g(t) \Gamma(\alpha) t^{\alpha}\right)$, where $E_{\alpha}$ is the Mittag-Leffler function defined by

$$
E_{\alpha}(z)=\sum_{k=0}^{\infty} \frac{z^{k}}{\Gamma(k \alpha+1)}, z \in C, \operatorname{Re}(\alpha)>0 .
$$

Lemma 2.17. [24] Let $x \in P C_{1-\gamma}(J, R)$ satisfies the following inequality

$$
|x(t)| \leq c_{1}+c_{2} \int_{0}^{t}(t-s)^{\alpha-1}|x(t)| d s+\sum_{0<t_{k}<t} \psi_{k}\left|x\left(t_{k}\right)\right|,
$$

where $c_{1}$ is a nonnegative, continuous and nondecreasing function and $c_{2}, \psi_{i}$ are constants. Then

$$
|x(t)| \leq c_{1}\left(1+\psi E_{\alpha}\left(c_{2} \Gamma(\alpha) t^{\alpha}\right)^{k} E_{\alpha}\left(c_{2} \Gamma(\alpha) t^{\alpha}\right) \text { for } t \in\left(t_{k} \cdot t_{k+1}\right]\right.
$$

where $\psi=\sup \left\{\psi_{k}: k=1,2,3, \ldots, m\right\}$.

Theorem 2.18. [5](Schaefer's Fixed Point Theorem) Let $K$ be a Banach space and let $N: K \rightarrow K$ be completely continuous operator. If the set $\{x \in K: x=\delta N x$ for some $\delta \in(0,1)\}$ is bounded, then $N$ has a fixed point.

Theorem 2.19. [5](Banach Fixed Point Theorem) Suppose $Q$ be a non-empty closed subset of a Banach space E. Then any contraction mapping $N$ from $Q$ into itself has a unique fixed point.

\section{Existence of at least one solution}

In this section, we investigate the existence and uniqueness of solution to the proposed problem. We need the following lemma to establish our main results.

Lemma 3.1. Let $f: J \times R \times R \rightarrow R$ be continuous. A function $x$ is a solution of the fractional integral equation

$$
x(t)=\frac{x_{a}}{\Gamma(\gamma)}(t-a)^{\gamma-1}+\frac{\sum_{0<t_{k}<t} \psi_{k}\left(x\left(t_{k}\right)\right)}{\Gamma(\gamma)}(t-a)^{\gamma-1}+\frac{1}{\Gamma(\alpha)} \int_{a}^{t}(t-s)^{\alpha-1} f(s, x(s), x(\lambda s)) d s
$$

if and only if $x$ is a solution of the problem (1.1).

Let us introduce the following assumptions which are used hereafter.

(H1) Let $f: J \times R \times R \rightarrow R$ be a continuous function and there exists a positive constant $\ell>0$, such that

$$
\left|f\left(t, x_{1}, x_{2}\right)-f\left(t, y_{1}, y_{2}\right)\right| \leq \ell\left(\left|x_{1}-y_{1}\right|+\left|x_{2}-y_{2}\right|\right), \text { for all } x_{1}, x_{2}, y_{1}, y_{2} \in R \text {. }
$$

(H2) Let $f: J \times R \times R \rightarrow R$ is completely continuous function and there exists a function $p \in L^{1}$ such that

$$
|f(t, x, y)| \leq p(t), \forall t \in J, x, y \in R .
$$

(H3) Let the functions $\psi_{k}: R \rightarrow R$ are continuous and there exists a constant $\ell_{k}^{*}>0$, such that

$$
\left|\psi_{k}(x)-\psi_{k}(y)\right| \leq \ell_{k}^{*}|x-y|, \text { for all } x, y \in R, k=1,2, \ldots, m \text {. }
$$

(H4) Let the functions $\psi_{k}: R \rightarrow R$ are continuous and there exists a constant $p^{*}>0$, such that

$$
\left|\psi_{k}(x)\right| \leq p^{*}(t), \text { for all } x \in R, k=1,2, \ldots, m .
$$

(H5) : There exists an increasing finctions $\varphi \in P C_{1-\gamma}\left[J, R^{+}\right]$and there exists $\lambda_{\varphi}>0$ such that for any $t \in J$,

$$
I^{\alpha} \varphi(t) \leq \lambda_{\varphi} \varphi(t) .
$$

Theorem 3.2 (Existence). Assume that [H1] - [H4] are satisfied. Then, Eq.(1.1) has at least one solution.

Proof. Consider the operator $N: P C_{1-\gamma}[J, R] \rightarrow P C_{1-\gamma}[J, R]$. The operator form of integral equation (3.1) is written as follows

$$
x(t)=N x(t),
$$

where

$$
N x(t)=\frac{x_{a}}{\Gamma(\gamma)}(t-a)^{\gamma-1}+\frac{\sum_{0<t_{k}<t} \psi_{k}\left(x\left(t_{k}\right)\right)}{\Gamma(\gamma)}(t-a)^{\gamma-1}+\frac{1}{\Gamma(\alpha)} \int_{a}^{t}(t-s)^{\alpha-1} f(s, x(s), x(\lambda s)) d s .
$$

First, we prove that the operator $N$ defined by (3.2) verifies the conditions of Theorem 2.18 .

Claim 1: The operator $N$ is continuous. Let $x_{n}$ be a sequence such that $x_{n} \rightarrow x$ in $P C_{1-\gamma}[J, R]$. Then for each $t \in J$,

$$
\begin{aligned}
\left|\left(N\left(x_{n}\right)(t)-N(x)(t)\right)(t-a)^{1-\gamma}\right| & \leq \frac{1}{\Gamma(\gamma)} \sum_{0<t_{k}<t}\left|\psi_{k}\left(x_{n}\left(t_{k}\right)\right)-\psi_{k}\left(x\left(t_{k}\right)\right)\right| \\
& +\frac{(t-a)^{1-\gamma}}{\Gamma(\alpha)} \int_{a}^{t}(t-s)^{\alpha-1}\left|f\left(s, x_{n}(s), x_{n}(\lambda s)\right)-f(s, x(s), x(\lambda s))\right| d s .
\end{aligned}
$$


since $f$ is continuous, then we have

$$
\left\|N\left(x_{n}\right)(t)-N(x)(t)\right\|_{P C_{1-\gamma}} \rightarrow 0 \text { as } n \rightarrow \infty .
$$

This proves the continuity of $N$.

Claim 2: The operator $N$ maps bounded sets into bounded sets in $P C_{1-\gamma}[J, R]$. Indeed, it is enough to show that for $r>0$, there exists a positive constant $l$ such that

$$
B_{r}=\left\{x \in P C_{1-\gamma}[J, R]:\|x\|_{P C_{1-\gamma}} \leq r\right\}
$$

we have $\|N(x)\|_{P C_{1-\gamma}} \leq l$.

$$
\begin{aligned}
& \mid(N x)(t)(t-a)^{1-\gamma \mid} \leq \frac{\left|x_{a}\right|}{\Gamma(\gamma)}+\frac{\sum_{0<t_{k}<t}\left|\psi_{k}\left(x\left(t_{k}\right)\right)\right|}{\Gamma(\gamma)}+\frac{(t-a)^{1-\gamma}}{\Gamma(\alpha)} \int_{a}^{t}(t-s)^{\alpha-1}|f(s, x(s), x(\lambda s))| d s \\
& \leq \frac{\left|x_{a}\right|}{\Gamma(\gamma)}+\frac{(t-a)^{\gamma-1} \sum_{0<t_{k}<t}\left|(t-a)^{1-\gamma} p^{*}(t)\right|}{\Gamma(\gamma)}+\frac{(t-a)^{1-\gamma}}{\Gamma(\alpha)} \int_{a}^{t}(t-s)^{\alpha-1}|p(s)| d s \\
& \leq \frac{\left|x_{a}\right|}{\Gamma(\gamma)}+\frac{m(t-a)^{\gamma-1}}{\Gamma(\gamma)}\left\|p^{*}\right\|_{P C_{1-\gamma}}+\frac{(t-a)^{1-\gamma}}{\Gamma(\alpha)}(t-a)^{\alpha+\gamma-1} B(\gamma, \alpha)\|p\|_{P C_{1-\gamma}} \\
& \leq \frac{\left|x_{a}\right|}{\Gamma(\gamma)}+\frac{m(b-a)^{\gamma-1}}{\Gamma(\gamma)}\left\|p^{*}\right\|_{P C_{1-\gamma}}+\frac{1}{\Gamma(\alpha)}(b-a)^{\alpha} B(\gamma, \alpha)\|p\|_{P C_{1-\gamma}} \\
&\|(N x)(t)\|_{P C_{1-\gamma}}:=l .
\end{aligned}
$$

That is $N$ is bounded.

Claim 3:The operator $N$ maps bounded sets into equicontinuous set of $P C_{1-\gamma}[J, R]$. Let $t_{1}, t_{2} \in J, t_{1}>t_{2}, B_{r}$ be a bounded set of $P C_{1-\gamma}[J, R]$ as in Claim 2, and $x \in B_{r}$. Then,

$$
\begin{aligned}
\left.\mid\left(t_{1}-a\right)^{1-\gamma}(N x)\left(t_{1}\right)-\left(t_{2}-a\right)^{1-\gamma}(N x)\left(t_{2}\right)\right) \mid & \leq\left|\frac{\sum_{0<t_{k}<t_{1}} \psi_{k}\left(x\left(t_{k}\right)\right)}{\Gamma(\gamma)}+\frac{\left(t_{1}-a\right)^{1-\gamma}}{\Gamma(\alpha)} \int_{a}^{t_{1}}\left(t_{1}-s\right)^{\alpha-1} f(s, x(s), x(\lambda s)) d s\right| \\
& -\left|\frac{\sum_{0<t_{k}<t_{2}} \psi_{k}\left(x\left(t_{k}\right)\right)}{\Gamma(\gamma)}-\frac{\left(t_{2}-a\right)^{1-\gamma}}{\Gamma(\alpha)} \int_{a}^{t_{2}}\left(t_{2}-s\right)^{\alpha-1} f(s, x(s), x(\lambda s)) d s\right| .
\end{aligned}
$$

As $t_{1} \rightarrow t_{2}$, the right hand side of the above inequality tends to zero. As a consequence of Claim 1 - Claim 3 together with Arzel $\ddot{a}$-Ascoli theorem, we can conclude that $N: P C_{1-\gamma}[J, R] \rightarrow P C_{1-\gamma}[J, R]$ is continuous and completely continuous.

It is continuous and bounded from Claim 1 - Claim 3. Now, it remains to show that the set

$$
\omega=\left\{x \in P C_{1-\gamma}[J, R]: x=\tau N(x), 0<\tau<1\right\}
$$

is bounded set.

Let $x \in \omega, x=\tau N(x)$ for some $0<\tau<1$. Thus for each $t \in J$. We have

$$
x(t)=\tau\left[\frac{x_{a}}{\Gamma(\gamma)}(t-a)^{\gamma-1}+\frac{\sum_{0<t_{k}<t} \psi_{k}\left(x\left(t_{k}\right)\right)}{\Gamma(\gamma)}(t-a)^{\gamma-1}+\frac{1}{\Gamma(\alpha)} \int_{a}^{t}(t-s)^{\alpha-1} f(s, x(s), x(\lambda s)) d s\right] .
$$

This shows that the set $\omega$ is bounded. As a consequence of Theorem 2.18, we deduce that $N$ has a fixed point which is a solution of problem (1.1).

Theorem 3.3. (Uniqueness) Assume that [H1] and [H3] are satisfied. If

$$
\rho=\left(\frac{m \ell^{*}}{\Gamma(\gamma)}(b-a)^{1-\gamma}+\frac{2 \ell}{\Gamma(\alpha)} B(\gamma, \alpha)(b-a)^{\alpha}\right)<1
$$

then, the Eq. (1.1) has a unique solution.

Proof. Consider the operator $N: P C_{1-\gamma}[J, R] \rightarrow P C_{1-\gamma}[J, R]$. The equivalent integral equation (3.1) which can be written in the operator form

$$
N x(t)=\frac{x_{a}}{\Gamma(\gamma)}(t-a)^{\gamma-1}+\frac{\sum_{0<t_{k}<t} \psi_{k}\left(x\left(t_{k}\right)\right)}{\Gamma(\gamma)}(t-a)^{\gamma-1}+\frac{1}{\Gamma(\alpha)} \int_{a}^{t}(t-s)^{\alpha-1} f(s, x(s), x(\lambda s)) d s .
$$


By Lemma 3.2, it is clear that the fixed points of $N$ are solutions of Eq. (1.1).

Let $x, y \in P C_{1-\gamma}[J, R]$ and $t \in J$, then we have

$$
\begin{aligned}
\left|(t-a)^{1-\gamma}(N x(t)-N y(t))\right| & \leq \frac{1}{\Gamma(\gamma)} \sum_{0<t_{k}<t}\left|\psi_{k}\left(x\left(t_{k}\right)\right)-\psi_{k}\left(y\left(t_{k}\right)\right)\right|+\frac{(t-a)^{1-\gamma}}{\Gamma(\alpha)} \int_{a}^{t}(t-s)^{\alpha-1}|f(s, x(s), x(\lambda s))-f(s, y(s), y(\lambda s))| d s \\
& \leq(t-a)^{1-\gamma} \frac{m \ell^{*}}{\Gamma(\gamma)}\|x-y\|_{P C_{1-\gamma}}+\frac{2 \ell(t-a)^{1-\gamma}}{\Gamma(\alpha)} B(\gamma, \alpha)(t-a)^{\alpha+\gamma-1}\|x-y\|_{P C_{1-\gamma}} \\
& \leq\left(\frac{m \ell^{*}}{\Gamma(\gamma)}(b-a)^{1-\gamma}+\frac{2 \ell}{\Gamma(\alpha)} B(\gamma, \alpha)(b-a)^{\alpha}\right)\|x-y\|_{P C_{1-\gamma}} \\
& =\rho\|x-y\|_{P C_{1-\gamma}} .
\end{aligned}
$$

This yields that $N$ has a unique fixed point which is solution of Eq. (1.1).

\section{U-H stability analysis}

In this section, we obtain stability results for the proposed problem.

Theorem 4.1. The assumptions [H1], [H3], [H5] and (3.3) hold. Then, Eq.(1.1) is generalized U-H-R stable.

Proof. Let $z$ be solution of inequality (2.5) and by Theorem 3.3 there $x$ is unique solution of the problem

$$
\begin{aligned}
D^{\alpha, \beta} x(t) & =f(t, x(t), x(\lambda t)), \\
\left.\Delta I^{1-\gamma} x(t)\right|_{t=t_{k}} & =\psi_{k}\left(x\left(t_{k}^{-}\right)\right), \\
I^{1-\gamma} x(a) & =I^{1-\gamma} z(a)=x_{a} .
\end{aligned}
$$

Then, we have

$$
x(t)=\frac{x_{a}}{\Gamma(\gamma)}(t-a)^{\gamma-1}+\frac{\sum_{0<t_{k}<t} \psi_{k}\left(x\left(t_{k}\right)\right)}{\Gamma(\gamma)}(t-a)^{\gamma-1}+\frac{1}{\Gamma(\alpha)} \int_{a}^{t}(t-s)^{\alpha-1} f(s, x(s), x(\lambda s)) d s
$$

By differentiating inequality (2.5), for each $t \in\left(t_{k}, t_{k+1}\right]$, we have

$$
\begin{gathered}
\left|z(t)-\frac{x_{a}}{\Gamma(\gamma)}(t-a)^{\gamma-1}-\frac{\sum_{0<t_{k}<t} \psi_{k}\left(z\left(t_{k}\right)\right)}{\Gamma(\gamma)}(t-a)^{\gamma-1}-\frac{1}{\Gamma(\alpha)} \int_{a}^{t}(t-s)^{\alpha-1} f(s, z(s), z(\lambda s)) d s\right| \\
\leq\left|\frac{\sum_{0<t_{k}<t} g_{k}}{\Gamma(\gamma)}(t-a)^{\gamma-1}+\frac{1}{\Gamma(\alpha)} \int_{a}^{t}(t-s)^{\alpha-1} \varphi(t) d s\right| \\
\leq m \varphi(t)+\lambda_{\varphi} \varphi(t) \\
\leq\left(m+\lambda_{\varphi}\right) \varphi(t) .
\end{gathered}
$$

Hence for each $t \in\left(t_{k}, t_{k+1}\right]$, it follows

$$
\begin{aligned}
|z(t)-x(t)| & \leq\left|z(t)-\frac{x_{a}}{\Gamma(\gamma)}(t-a)^{\gamma-1}-\frac{\sum_{0<t_{k}<t} \psi_{k}\left(x\left(t_{k}\right)\right)}{\Gamma(\gamma)}(t-a)^{\gamma-1}-\frac{1}{\Gamma(\alpha)} \int_{a}^{t}(t-s)^{\alpha-1} f(s, x(s), x(\lambda s)) d s\right| \\
& \leq\left|z(t)-\frac{x_{a}}{\Gamma(\gamma)}(t-a)^{\gamma-1}-\frac{\sum_{0<t_{k}<t} \psi_{k}\left(z\left(t_{k}\right)\right)}{\Gamma(\gamma)}(t-a)^{\gamma-1}-\frac{1}{\Gamma(\alpha)} \int_{a}^{t}(t-s)^{\alpha-1} f(s, z(s), z(\lambda s)) d s\right| \\
& +\frac{\sum_{0<t_{k}<t}\left|\psi_{k}\left(z\left(t_{k}\right)\right)-\psi_{k}\left(x\left(t_{k}\right)\right)\right|}{\Gamma(\gamma)}(t-a)^{\gamma-1}+\frac{1}{\Gamma(\alpha)} \int_{a}^{t}(t-s)^{\alpha-1}|f(s, x(s), x(\lambda s))-f(s, x(s), x(\lambda s))| d s \\
& \leq\left(m+\lambda_{\varphi}\right) \varphi(t)+\frac{m \ell^{*}}{\Gamma(\gamma)}(t-a)^{\gamma-1}|z(t)-x(t)|+\frac{2 \ell}{\Gamma(\alpha)} \int_{a}^{t}(t-s)^{\alpha-1}|z(t)-x(t)| d s
\end{aligned}
$$

By Lemma 2.17, there exists a constant $K>0$ independent of $\lambda_{\varphi} \varphi(t)$ such that

$$
|z(t)-x(t)| \leq K\left(m+\lambda_{\varphi}\right) \varphi(t):=C_{f, \varphi} \varphi(t) .
$$

Thus, Eq.(1.1) is generalized U-H-R stable. 


\section{References}

[1] S. Harikrishnan, Rabha W. Ibrahim, K. Kanagarajan, On y-Hilfer Fractional Differential Equation with Complex Order, Universal Journal of Mathematics and Applications(1) (2018) 33-38 Universal Journal of Mathematics and Applications

[2] M. I. Abbas, Ulam stability of fractional impulsive differential equations with Riemann-Liouville integral boundary conditions, J. Contemp. Mathemat. Anal., 50, (2015), 209-219.

[3] K. Balachandran, S. Kiruthika, J.J. Trujillo, Existence of solutions of Nonlinear fractional pantograph equations, Acta Math. Sci., 33B,(2013),1-9.

[4] K.M. Furati, M.D. Kassim and N.e-. Tatar, Existence and uniqueness for a problem involving Hilfer fractional derivative, Comput. Math. Appl., 64, (2012), 1616-1626.

[5] A. Granas, and J. Dugundji, Fixed Point Theory, Springer-Verlag, New York, 2003.

[6] K. Guan, Q. Wang, X. He, Oscillation of a pantograph differential equation with impulsive perturbations, Appl. Math. Comput., 219, (2012), 3147-3153.

[7] S. Harikrishnan, K. Kanagarajan and E. M. Elsayed, Existence and stability results for langevin equations with Hilfer fractional derivative, Res. Fixed Point Theory Appl., (2018), 10 pages.

[8] D.H. Hyers, On the stability of the linear functional equation, Proc. Natl. Acad. Sci., 27, (1941), $222-224$.

[9] R. Hilfer, Applications of Eractional Calculus in Physics, World scientific, Singapore, 1999.

[10] A. Iserles, On the generalized pantograph functional differential equation, European J. Appl. Math., 4, (1993), 1-38.

[11] R.W. Ibrahim, Ulam-Hyers stability for Cauchy fractional differential equation in the unit disk, Abstract Appl. Anal., (2012), 10 pages.

[12] R. W. Ibrahim, Generalized Ulam-Hyers stability for fractional differential equations, Int. J. Math., 23(5), (2012), 9 pages.

[13] A. A. Kilbas, H.M. Srivastava and J. J. Trujillo, Theory and applications of fractional differential equations, Amsterdam: Elsevier,2006.

[14] V. Lakshmikantham, D. D. Bainov and P. S. Simeonov, Theory of Impulsive Differential Equations, World scientific, Singapore, 1989.

[15] X. Liu and Y. Li, Some antiperiodic boundary value problem for nonlinear fractional impulsive differential equations, Abst. Appl. Anal., (2014), 10

[16] Z. Luo and J. Shen, Global existence results for impusive functional differential equation, J. Math. Anal. Appl., 323(1), (2006), 644-653.

[17] I. Podlubny, Fractional Differential equation, Academic Press, San Diego, 1999.

[18] A. M. Samoilenko and N. A. Perestyuk, Impulsive differential equations, World scientific, Singapore(1995).

[19] D. Vivek, K. Kanagarajan and S. Sivasundaram, Dynamics and stability of pantograph equations via Hilfer fractional derivative, Nonlinear Stud., 23(4), (2016),685-698.

[20] D. Vivek, K. Kanagarajan and E. M. Elsayed, Some existence and stability results for Hilfer-fractional implicit differential equations with nonlocal conditions, Mediterr. J. Math., 15:15, (2018).

[21] D. Vivek, K. Kanagarajan and S. Sivasundaram, Dynamics and stability results for Hilfer fractional type thermistor problem, Fractal Fract., 1(1), (2017), $1-14$.

[22] D. Vivek, K. Kanagarajan, S. Harikrishnan, Existence and uniqueness results for pantograph equations with generalized fractional derivative, Journal of Nonlinear Analysis and Application, 2, (2017), 105-112.

[23] J. Wang, L. Lv and Y. Zhou, Ulam stability and data dependence for fractional differential equations with Caputo derivative, Electron. J. Qual. Theory Differ. Equ., 63, (2011), 1-10.

[24] J. Wang, Y. Zhou and M. Fec̈kanc, Nonlinear impulsive problems for fractional differential equations and Ulam stability, Comp. Math. Appl., 64, (2012), $3389-3405$.

[25] H. Ye, J. Gao and Y. Ding, A generalized Gronwall inequality and its application to a fractional differential equation, J. Math. Anal. Appl., 328(2), (2007), 1075-1081. 